\title{
Mixin-based Inheritance
}

\author{
Gilad Bracha* \\ Department of Computer Science \\ University of Utah \\ Salt Lake City, UT 84112
}

\author{
William Cook \\ Hewlett-Packard Laboratories \\ 1501 Page Mill Road \\ Palo Alto, CA 94303-0969
}

\section{Abstract}

The diverse inheritance mechanisms provided by Smalltalk, Beta, and CLOS are interpreted as different uses of a single underlying construct. Smalltalk and Beta differ primarily in the direction of class hierarchy growth. These inheritance mechanisms are subsumed in a new inheritance model based on composition of mixins, or abstract subclasses. This form of inheritance can also encode a CLOS multiple-inheritance hierarchy, although changes to the encoded hierarchy that would violate encapsulation are difficult. Practical application of mixin-based inheritance is illustrated in a sketch of an cxtension to Modula-3.

\section{Introduction}

A variety of inheritance mechanisms have been developed for object-oriented programming languages. These systems range from classical Smalltalk single inheritance [8], through the safer prefixing of Beta $[12,10]$, to the complex and powerful multiple inheritance combinations of CLOS $[6,9]$. These languages have similar object models, and also share the view that inheritance is a mechanism for incremental programming. However, they differ widely in the kind of incremental changes supportcd.

In Smalltalk, subclasses can add additional methods or replace existing methods in the parent class. As a result, there is no necessary relationship between the behavior of instances of a class and the instances of its subclasses. The subclass methods can invoke any of the original superclass methods via super.

In Beta, a subpattern (subclass) definition is viewed as an extension of a previously defined prefix pattern. As

"Supported by grant CCR-8704778 from the National Science Foundation.

Permission to copy without fee all or part of this material is granted provided that the copies are not made or distributed for direct commercial advantage, the ACM copyright notice and the title of the publication and its date appear, and notice is given that copying is by permission of the Association for Computing Machinery. To copy atherwise, or to republish, requires a fee and/or specific permission.

( 1990 ACM 089791-411-2/90/0010-0303...\$1.50 in Smalltalk, new methods may be defined. However, prefix methods cannot be replaced; instead, the prefix may use the command inner to invoke the extended method code supplied by the subpattern. Given that the code in a prefix is executed in any of its extensions, Beta enforces a degree of behavioral consistency between a pattern and its subpatterns.

The underlying mechanism of inheritance is the same for Beta and Smalltalk [3]. The difference between them lics in whether the extensions to an existing definition have precedence over and may refer to previous definitions (Smalltalk), or the inherited definition has precedence over and may refer to the extensions (Beta). This model shows that Beta and Smalltalk have inverted inheritance hierarchies: a Smalltalk subclass refers to its parent using super just as a Beta prefix refers to its subpatterns using inner.

In the Common Lisp Object System (CLOS) and its predecessor, Flavors [13], multiple parent classes may be merged during inheritance. A class's ancestor graph is linearized so that each ancestor occurs only once [7]. With standard method combination for primary methods, the function call-next-method is used to invoke the next method in the inheritance chain.

CLOS supports mixins as a useful technique for building systems out of mixable attributes. A mixin is an abstract subclass; i.e. a subclass definition that may be applied to different superclasses to creale a related family of modified classes. For example, a mixin might be defined that adds a border to a window class; this mixin could be applied to any kind of window to create a bordered-window class. Semantically, mixins are closely related to Beta prefixes.

Linearization has been criticized for violating encapsulation, because it may change the parent-child relationships among classes in the inheritance hierarchy $[16,17]$. But the mixin technique in CLOS depends directly upon linearization and modification of parentchild relationships. Rather than avoid mixins because they violate encapsulation, we argue that linearization is an implementation technique for mixins that obscures their true nature as abstractions.

By modest generalization of the inheritance models 
in Smalltalk and Beta, a form of inheritance based on composition of mixins is derived. Mixin-based inheritance supports both the flexibility of Smalltalk and the security of Beta. It also supports the direct encoding of CLOS multiple inheritance hierarchies without duplication of subclass definitions. However, since the hierarchy is encoded as an explicit collection of linearized inheritance chains rather than as a single inheritance graph, some changes to the hierarchy (especially if they might violate Snyder's notion of encapsulation) cannot easily be made.

Section 2 discusses the single-inheritance languages Smalltalk and Beta and shows that they support very different uses of a single underlying construct. Section 3 analyzes multiple inheritance and linearization in CLOS, with special focus on support for mixins. Section 4 presents a generalized inheritance mechanism that supports the style of inheritance in Beta, Smalltalk, and CLOS, with explicit support for mixins. In Section 5 we sketch an extension to Modula-3 that illustrates the use of generalized inheritance. Finally, Section 6 summarizes our conclusions.

\section{Single Inheritance Languages}

\subsection{Smalltalk Inheritance}

Inheritance in Smalltalk is a mechanism for incremental derivation of classes. Smalltalk inheritance was adapted from Simula $[5,14]$, and serves as the prototypical inheritance mechanism. The primary subtlety in the process of inheritance is the interpretation of the special variables self and super. Self represents recursion, or self-reference, within the object instance being defined. The interpretation of self has been addressed in previous work $[3,4,15]$; in this paper we focus on the interpretation of super. Consider the following pair of Smalltalk classes.

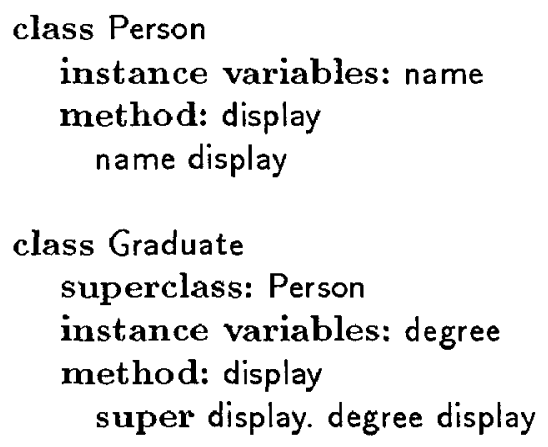

The class Person defines a name field and a method for displaying the name. The subclass Graduate extends the display method to include the person's academic degree. For example, a graduate with name "A. Smith" and degree "Ph.D." would respond to the display method by invoking the Graduate display method, which invokes the Person display method using super display to display the name, and then displays the degree. The net effect would be to print "A. Smith Ph.D.". It would also be possible to prefix the name, as in the case of titles like "Dr.", by printing the title before calling super.

The subclass Graduate specifies only how Graduates differ from Persons [19]. This difference may be indicated explicitly as a delta, or set of changes. In this case the set of changes is simply the new display method. The original definition is also just a display method. When combined, the new display method replaces the original.

To formalize this process, objects are represented as records whose fields contain methods $[1,15,18,3]$. The cxpression $\left\{a_{1} \mapsto v_{1}, \cdots, a_{n} \mapsto v_{n}\right\}$ represents a record with fields $a_{1}, \ldots, a_{n}$ and associated values $v_{1}, \ldots, v_{n}$. The expression r. $a$ represents selection of field $a$ from a record $r$. Record combination is a binary operator, $\oplus$, that forms a new record with the fields from its two arguments, where the value is from the left argument in case the same field is present in both records. For example, $\{a \mapsto 3, b \mapsto$ 'x' $\} \oplus\{a \mapsto$ true, $c \mapsto 8\}$ replaces the right value of $a$ to produce $\left\{a \mapsto 3, b \mapsto\right.$ ' $\left.\mathrm{x}^{\prime}, c \mapsto 8\right\}$,

To interpret super, it is necessary for the delta, or modifications, to access the original method inherited from Person. This is achieved by supplying the parent class methods as a pararneter to the delta. The resulting inheritance mechanism is an asymmetric combination of a. parametric delta $\Delta$ and a parent specification $P$ :

$$
C=\Delta(P) \oplus P .
$$

This definition is a form of single inheritance: $P$ refers to the inherited parent while $\Delta$ is an explicit set of changes. The two occurrences of $P$ do not indicate that it is instantiated twice, but that its information is used in two contexts: for the interpretation of super and to provide methods for the subclass. Suppressing the interpretation of hidden instance variables, the example above has the following form.

$$
\begin{aligned}
& P=\{\text { display } \mapsto \text { name.display }\} \\
& \Delta(s)=\{\text { display } \mapsto s \text { display, degree.display }\} \\
& \Delta(P)=\{\text { display } \mapsto \text { name.display, degree.display }\}
\end{aligned}
$$

Although deltas were introduced to make specification of the inheritance mechanism more clear, deltas are not independent elements of a Smalltalk program; they cannot stand on their own and are always part of a subclass definition, which has an explicit parent class.

In Smalltalk a subclass of Person may completely replace the display method with, for example, a routine that displays the time of day. In Smalltalk inheritance, the subclass is in control: there is no way to define Person so that it forces subclasses to invoke its display method as part of their display operation. 


\subsection{Beta Inheritance}

Inheritance in Beta is designed to provide security from replacement of a method by a completely different method. Inheritance is supported in Beta by prefixing of definitions. Beta employs a single definitional construct, the pattern, to express types, classes and methods. As this generality can be confusing, we use a simpler syntax that distinguishes among the different roles ${ }^{1}$. The example given above is easily recoded in Beta:

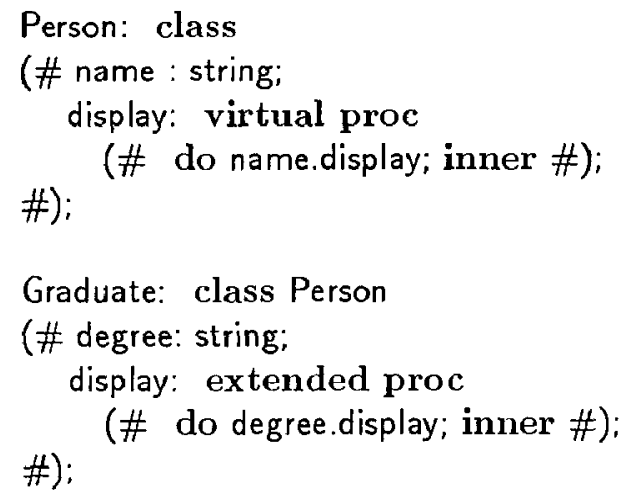

The definition of Graduate is said to be prefixed by Person. Person is the superpattern of Graduate, which, correspondingly, is a subpattern of Person. Display is declared to be virtual, which means that it may be extended in a subpattern. This does not mean that, it may be arbitrarily redefined, as in most object-oriented languages.

The behavior of the display method of a Person is to display the name and then perform the inner statement. For a plain Person instance, which has no inner behavior, the inner statement is a null operation (i.e. skip or no-op). When a subpattern of Person is defined, the inner statement will execute the corresponding display method in the subpattern.

The subpattern Graduate extends the behavior of the Person display method by supplying inner behavior. For a Graduate instance $G$, the initial effect of G.display is the same as for a Person: the original method from Person is exccuted. $\Lambda \mathrm{ftcr}$ the name is displaycd, the inner procedure supplied by Graduate is executed to display the graduatc's degree. The use of inner within Graduate is again interpreted as a no-op. It only has an effect if the display method is extended by a subpattern of Graduate. It is impossible to arrange for printing a title, like "Dr.", before the name by inheriting Person; this is because the choice to invoke inner after the name has been built into the Person display method. In Beta prefixing, the prefix controls the behavior of the result.

The interpretation of the Person pattern is as a parametric definition of attributes, $P^{\prime}$. The parameter rep-

\footnotetext{
${ }^{1}$ This syntax is used by the implementors of Beta for tutorial purposes [11].
}

resents any inner definitions supplied by subpatterns. For an instance of Person, the inner part of $P^{\prime}$ is bound to the record of null methods: $P^{\prime}(\emptyset)$.

$\Lambda$ subpattern specifies additional attributes which may also refer to any further inner behavior in later subpatterns. If the attributcs defined in the subpattern are specified by $\Delta^{\prime}$, then the result of prefixing by $P^{\prime}$ is the following composition:

$$
C^{\prime}(\text { inner })=P^{\prime}\left(\Delta^{\prime}(\text { inner }) \oplus \text { inner }\right) \oplus \Delta^{\prime}(\text { inner })
$$

This means that the interpretation $C^{\prime}$ of the subpattern, when supplied an inner parameter, is the result of combining the superpattern $P^{\prime}$ specification with the changes specified by $\Delta^{\prime}$. By applying $P^{\prime}$ to $\Delta^{\prime}$ (inner) $\oplus$ inner, the inner specification of $P^{\prime}$ is bound to the fields of the subpattern combined with any further fields supplied by later subpatterns. The prefix methods take precedence over the suffix. In the example above, the equation for $C^{\prime}$ is greatly simplified by examining the actual uses of inner:

$$
\begin{aligned}
P^{\prime}(i)=\{\text { display } \mapsto \text { name.display, } i \text {.display }\} \\
\Delta^{\prime}(i)=\{\text { display } \mapsto \text { degree.display, } i \text { display }\} \\
C^{\prime}(i)=\{\text { display } \mapsto \text { name.display, } \\
\text { degree.display, } \\
\text { i.display }\}
\end{aligned}
$$

This formulation does not directly encode the restriction that inner within a method $m$ can refer only to the suffix method named $m$. In this sense inner is less general than Smalltalk's super construct, but the restriction is justified by the desire for security. An alternative formalization that captures this restriction involves representing each method as a function of its inner behavior [3]. Prefixing is then defined as combination of records such that duplicated fields are composed. Before calling a method if must be applied to a null command so that inner will have no effect. The resulting formalism is equivalent to the one given above, under the condition that the fields of $P^{\prime}$ and $\Delta^{\prime}$ only accss corresponding fields of inner.

\subsection{Comparing Smalltalk and Beta}

The inheritance mechanisms of Smalltalk and Beta are different orientations of a common undcrlying mechanism. The underlying mechanism is a non-associative binary operator, $D$, that pcrforms application of super/inner and combination of attributes.

$$
\Delta \triangleright P=\Delta(P) \oplus P
$$

The relationship between Beta and Smalltalk is demonstrated by comparing the interpretations of inheritance in the two languages. The behavior of a subclass instance can be compared concisely in this framework. 

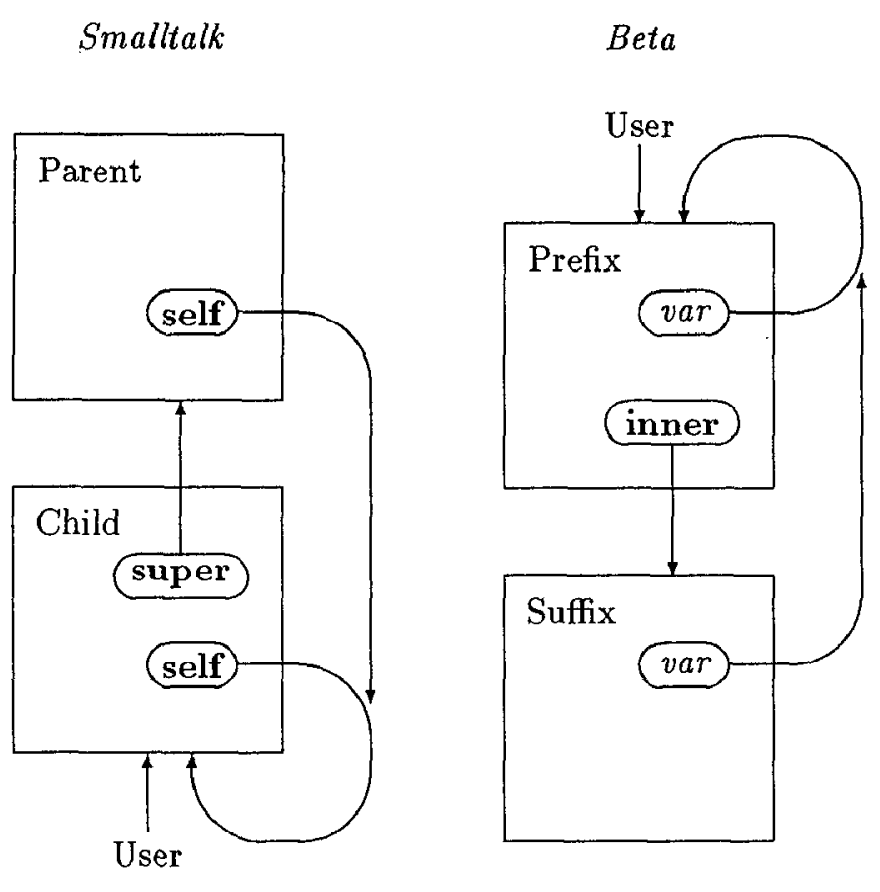

Figure 1: Inverse hierarchies in Smalltalk and Beta.

$$
\begin{aligned}
C & =\Delta \triangleright P & & \text { Smalltalk } \\
C^{\prime}(\emptyset) & =P^{\prime} \triangleright \Delta^{\prime}(\emptyset) & & \text { Beta }
\end{aligned}
$$

In these equations, $\Delta$ represents the new explicit information supplied by the subclass/subpattern, while $P$ represents the original attributes contributed by the superclass/superpattern. The combination operator $D$ favors values from its left argument in case of a duplicate attribute.

It is clear that the mechanism of inheritance is the same; only the direction of growth is different. In Smalltalk the new attributes are favored and may replace inherited ones; in Beta the original attributes are favored. Beta inheritance works in the opposite direction from inheritance in most object oriented languages, due to this role reversal between superpatterns/subpatterns and subclasses/superclasses. Figure 1 show this inversion by illustrating the semantic relationships in Smalltalk and Beta when a superclass is placed above one of its subclasses. The figure includes the interpretation of self-reference, which is implicit in Beta variable (var) references [3]. Neither direction of inheritance is able to express the other, and each has its advantages and disadvantages.

\section{Multiple Inheritance and Mixins}

\subsection{CLOS Inheritance}

CLOS supports a rich mechanism for multiple inheritance. Although there are several significant aspects of CLOS inheritance, we focus only on standard method combination and primary methods. Here is the example given above, recoded in CLOS.

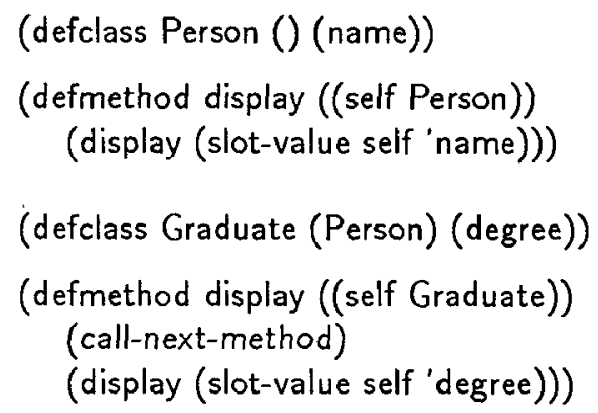

The defclass construct includes the name of the new class, a list of its superclasses, and a list of its instance variables. The argument list of the defmethod form defines the class on which the method is defined. Simple but effective method combination is supported by callnext-method, which plays the role of super in Smalltalk. But like inner in Beta, call-next-method provides access only to the next method in the inheritance chain with the same message selector.

A CLOS class may inherit from more than one parent. As a result, a given ancestor may be inherited more than once. For example, the following classes result in Person being inherited twice by Research-Doctor.

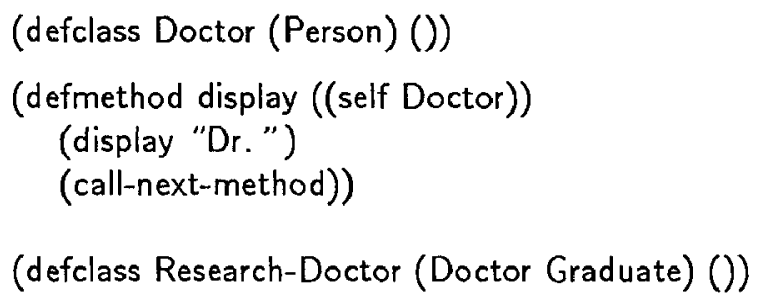

If care is not taken, the display method of Person will be executed twice, and a Research-Doctor will display as "Dr. A. SmithA. Smith Ph.D.". To remedy this situation, CLOS linearizes the ancestor graph of a class to produce an inheritance list in which each ancestor occurs only once. The graph of ancestors of ResearchDoctor is linearized to Research-Doctor, Doctor, Graduate, Person. This also solves the problem of method invocation order, because ancestor classes are placed in a linear order.

Each collection of method definitions may invoke methods later in the linearized sequence via call-nextmethod. If the specification of parents $P_{1}, \ldots, P_{n}$ is 
given by $\Delta_{1}, \ldots, \Delta_{n}$, then the interpretation $C$ of the subclass is defined by iteration of the inheritance operator over the list.

$$
C=\Delta_{1} \triangleright\left(\Delta_{2} \triangleright\left(\cdots \triangleright\left(\Delta_{n} \triangleright \emptyset\right)\right)\right)
$$

Each specification in the list is applied to the result of the previous specification and combined with it. The more complex method combination mechanisms of CLOS can also be modeled in this framework. For example, if before and after methods were distinguished then the base class, whose methods would be called after all other methods, could arrange for the last before method to call the primary method, and the last primary method to call the after method.

The process of linearization has been criticized for violating encapsulation [17]. One argument is that the relationship between a class and its declared parents may be modified during linearization. This is demonstrated by the example above, where in the linearization the class Graduate is placed between Doctor and Person, in contradiction of the explicit declaration of Doctor that it inherits directly from Person. Only by being aware of the entire class hierarchy can the programmer foresee this.

Using linearization, a CLOS multiple inheritance hierarchy is reduced to a collection of inheritance chains, each of which can be interpreted using single inheritance. However, a slight change to the original CLOS hierarchy may result in a very different collection of inheritance chains. This is especially true if the changes violate Snyder's notion of encapsulation, as when a base class is factored into two classes, because one of the new factors may interact with other classes during linearization. A less severe problem is that a given class may occur in many chains, so if the collection was implemented in a single-inheritance language, subclasses would have to be duplicated. In order to eliminate this duplication, the single-inheritance model must be generalized to allow explicit naming and reuse of the deltas defined by subclasses.

\subsection{Mixin Programming}

In this section we discuss a common programming technique used in CLOS, called mixins. A mixin is an abstract subclass that may be used to specialize the behavior of a variety of parent classes. It often does this by defining new methods that perform some actions and then call the corresponding parent methods. Mixins are very similar to the deltas introduced informally in Section 2.1. For example, the notion of a graduate degree as part of a name can be written as an independent mixin.

$$
\text { (defclass Graduate-mixin () (degree)) }
$$

\section{(defmethod display ((self Graduate-mixin)) \\ (call-next-method) \\ (display (slot-value self 'degree)))}

This example illustrates a characteristic of mixins: they invoke call-next-method even though they do not appear to have any parents. This would obviously lead to an error if an instance of a mixin were created. Linearization places the mixin into an inheritance chain before other classes that support the method. This occurs in the new definition of Graduate: because Graduatemixin is listed before Person, the Person display method will be invoked by Graduate-mixin display.

\section{(defclass Graduate (Graduate-mixin Person) ())}

In CLOS, mixins are simply a coding convention and have no formal status. Although locally unbound uses of call-next-method are a clear indication that a class is a mixin, the concept has no formal definition, and any class could be used as a mixin if it contributes partial behavior.

Using Graduate-mixin it is now possible to define different kinds of classes that have "graduated" behavior. In this example, the guard dog might have an obedience school degree.

\section{(defclass Guard-Dog (Graduate-mixin Dog) ())}

Neither Smalltalk nor Beta fully support mixins. In Smalltalk, the effect of a mixin can be achieved by explicitly creating subclasses and copying the mixin code into the subclass, preventing code-sharing and abstraction. In Beta, an individual class closely resembles a mixin. However, it cannot be attached to independently-defined classes. Instead, the client class must be built with the mixin as a prefix. If a family of mixed versions of a given class is needed, then the entire class must be copied for each prefixed mixin. Thus, in Smalltalk the mixin must be copied, while in Beta the base class must be copied. This is consistent with our analysis of the direction of growth in Beta and Smalltalk.

Mixin programming takes advantage of multiple inheritance in a subtle and unintuitive way: mixins depend upon linearization to place them in an appropriate location in the inheritance chain and to insert other classes between the mixin and its parents. When mixins are viewed as abstract subclasses, or class definitions parameterized by their parents, it is clear that linearization plays the role of application, by binding the mixin's formal parent parameter to a specific class. This process of abstraction and application can be made more explicit by generalizing the inheritance mechanism common to Smalltalk and Beta. 


\section{Inheritance as Composition of Mixins}

Mixins are the basis for a compositional inheritance mechanism that generalizes Smalltalk and Beta, while supporting the encoding of an encapsulated version of a CLOS multiple inheritance hierarchy. The basic idea of the generalization is to take mixins as the primary definitional construct. Inheritance is then formulated as composition of mixins. New attributes may be composed in either the Smalltalk or Beta style (either overwriting or extending). Since mixins and composition are explicit, there is no need for implicit linearization: a programmer would explicitly select the order of all mixin components. If a component is composed more than once it will appear as multiple copies in the result; duplication is avoided by explicitly applying two components to a shared parent.

The mixin composition operator, $\star$, is the Beta inheritance operator, but is used in a slightly more general form. Mixin composition takes two mixins as parameters, and returns a new mixin as a result.

$$
M_{1} \star M_{2}=\operatorname{fun}(i) M_{1}\left(M_{2}(i) \oplus i\right) \oplus M_{2}(i)
$$

In case of conflict, * gives priority lo the first parameter. In $M_{1}$, super/inner is bound during the inheritance operation to $M_{2}$. In $M_{2}$, super/inner is bound to the formal parameter $i$ of the result. Assuming the basic attribute combination operator $\oplus$ is associative, $\star$ is associative. In addition, if $\oplus$ were commutative, then * would be commutative.

Ordinary classes are viewed as degenerate mixins that do not make use of their inner/super parameter. Mixins thereby generalize Smalltalk classes, Beta patterns and CLOS style mixins. Abstract classes are viewed as mixins that refer to fields not defined in self. A mixin is complete if it does not refer to its parent parameter, and defines all fields that it refers to in itself. Otherwise, it is partial. Only complete mixins may be instantiated meaningfully.

\section{Application to an Existing Language}

\subsection{Choice of Language}

We have chosen Modula-3 [2] as a basis for an extension incorporating mixin-based inheritance. Modula-3 is well suited for such an extension, because it supports single inheritance and is strongly typed. Single inheritance naturally generalizes to mixin-based inheritance. Strong typing provides a framework in which mixins can be used safely and efficiently.

\subsection{Modula-3 Inheritance}

Modula-3 supports inheritance via object types. Object types are roughly analogous to classes in most objectoriented languages. An example of object types in Modula- 3 is

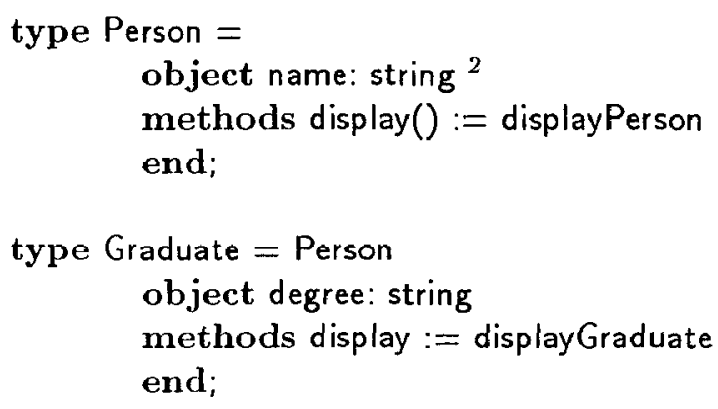

procedure displayPerson(self: Person) = begin

self.name display();

end displayPerson;

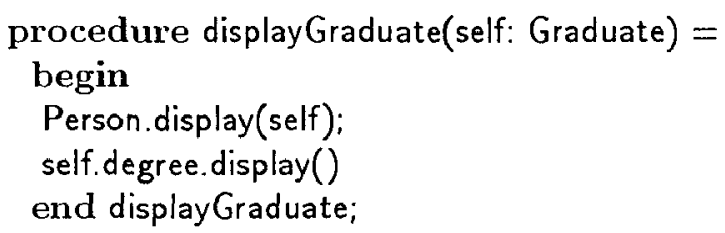

In the example, Person defines an instance variable name and a method display. The method is defined by providing a name, followed by a signature, or formal parameter list. In this case, the signature is empty. The method is then assigned a value, which is a separately defined procedure, displayPerson. If $\circ$ is an object of type Person, o.display () is interpreted as displayPerson(o).

The definition of Graduate has two parts: A preexisting definition, Person, and a modification given by the object ... methods ... end clause. Graduate is a subtype of Person, which is its supertype. Graduate inherits from Person, but includes a method override for display. The method override names the method being overridden, and then assigns a new value to it, namely displayGraduate. A signature is not given, since it will always be identical to the signature of the corresponding method in the supertype. The overridden methods of Person may be referred to by Graduate through the syntax Person.methodname. This is similar to super in Smalltalk, but more general.

An object ... methods ... end clause corresponds to the notion of delta discussed above. As in Smalltalk, deltas may not be defined independently of a parent. The following section presents an extension, whereby such deltas become independent constructs.

\footnotetext{
${ }^{2}$ Modula-3 uses TEXT for character

strings. However, we will assume that string has been defined.
} 


\subsection{Extending Modula-3}

We extend Modula-3 by generalizing object types to mixins. A mixin may be an explicit modification, of the form object ... methods ... end. Alternately, a mixin may be the result of combining two previously defined mixins.

$$
\begin{aligned}
& \text { Mixin }=\text { object } \ldots \text { methods } \ldots \text { end } \mid \\
& \text { Mixin }_{1} \star \text { Mixin }_{2}
\end{aligned}
$$

The concrete syntax used in the examples below, differs from the notation used until now in three respects. First, the order of the operands of the mixin operator . is reversed, so that priority is given to the right hand operand. Second, the mixin operation is not written explicitly, but is implicit between each pair of mixins in a mixin definition. Finally, an optional super clause is added to modifications. The first two changes reflect existing Modula-3 syntax, where a modification is written to the right of a base definition, with no composition operator in between. Adopting these changes helps make the extension upwardly compatible. The third change is for typechecking purposes, as explained below. The resulting syntax is

$$
\begin{aligned}
\text { Mixin' }= & \text { object } \ldots \text { methods } \ldots \text { end } \mid \\
& \text { object } \ldots \text { methods } \ldots \text { super } \ldots \text { end } \mid \\
& \text { Mixin' } \text { Mixin }_{1}^{\prime} .
\end{aligned}
$$

The following is equivalent to the CLOS mixin example given above.

$$
\begin{aligned}
& \text { type GraduateMixin = } \\
& \text { object degree: string } \\
& \text { methods display := displayGraduateMixin } \\
& \text { super display }():=\text { No_Op } \\
& \text { end; }
\end{aligned}
$$

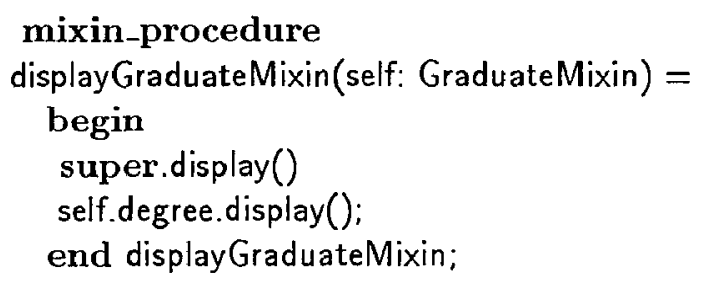

Since GraduateMixin is defined independently of any parent, the signature of display cannot be inferred, and must be given in a special super clause. Similarly, display's overridden value is not known, but may be assigned a default. In this case, the default value is No_Op, a procedure that will work on any type, since it is defined on root, the root of the type hierarchy. DisplayGraduateMixin refers to the overridden display method through the pseudo-variable super, using the syntax super.methodname. Procedures that reference super are distinguished, using the keyword mixin_procedure.

In the code above, GraduateMixin plays a role similar to a subclass in Smalltalk. Reversing GraduateMixin's position in the definition of Graduate reverses its role to that of a Beta subpattern. This is illustrated below, where PersonMixin functions as superpattern.

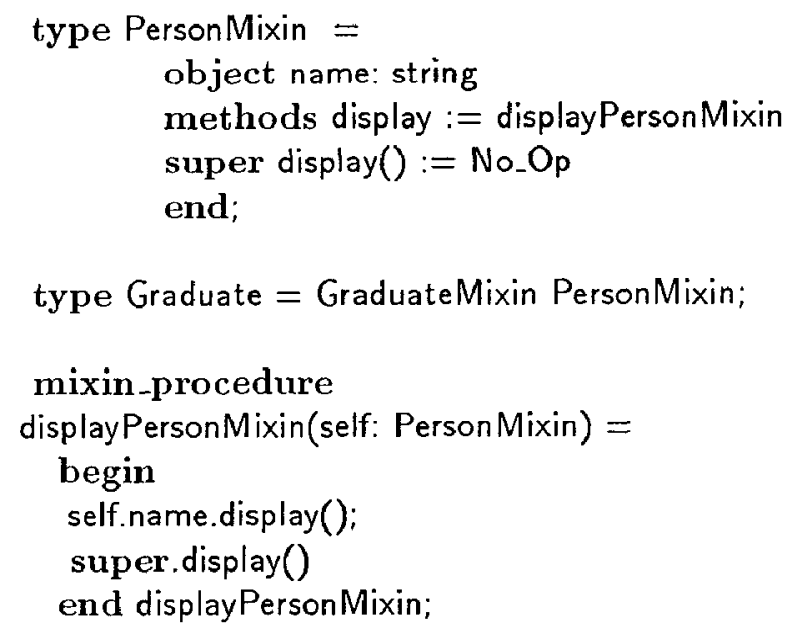

PersonMixin is in control, when combined with GraduateMixin. Graduate display() invokes displayPersonMixin, where super.display() calls displayGraduateMixin. In displayGraduateMixin super.display will use the default value, No_Op, corresponding to an empty inner clause in Beta.

The examples above have an important advantage over their Smalltalk and Beta counterparts; all parts of the definition can be reused, without being textually copied.

As a final example, we recode our earlier CLOS multiple inheritance example:

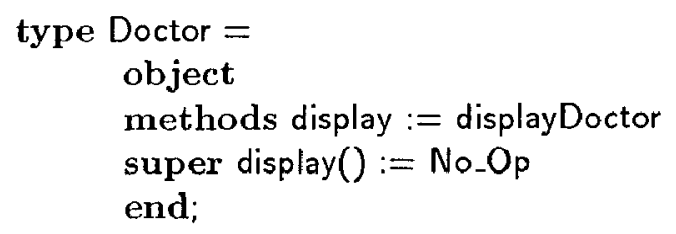

type ResearchDoctor $=$ Person Mixin GraduateMixin Doctor;

mixin_procedure displayDoctor(self:Doctor) $=$ begin

display( "Dr. "):

super.display()

end displayDoctor; 
Note how the linear sequence of definitions is given explicitly, without reliance on linearization.

\subsubsection{Typing}

This section presents the typing rules for mixins in the Modula-3 extension. The typing of mixins has not been addressed in prior work, since mixins have not been introduced into a strongly typed language before.

Type identity is defined as in Modula-3. Two types are identical iff their expanded definitions are identical. The subtyping relation on mixins, $T \ll S$ (read $T$ is a subtype of $S$, or $S$ is a supertype of $T$ ) is defined as follows:

1. object ... end $\ll$ root. All mixins are subtypes of root.

2. If $T_{1}=T_{2} T_{3}$ then $T_{1} \ll T_{2}$ and $T_{1} \ll T_{3}$, where the $=$ sign denotes type identity.

$3 . \ll$ is reflexive and transitive.

For example ResearchDoctor $\ll$ Doctor, as well as ResearchDoctor $\ll$ GraduateMixin, ResearchDoctor $\ll$ PersonMixin. What is less obvious is that if

PGMixin = PersonMixin GraduateMixin;

then ResearchDoctor «PGMixin. This follows from the fact that ResearchDoctor = PGMixin Doctor by the definition of type identity. Recall that the mixin combination operator, $\star$, is associative. This is reflected in the subtyping rules.

Additional rules for mixin composition include :

- A method should be mentioned in the super clause of a type, if it has been overridden. In practice, a method override may be omitted from the super clause, if its signature can be inferred from context. An example might be the definition of Graduate in section 5.2. This exception is made for compatibility with existing Modula-3 code.

- The pseudo-variable super may only be used in procedures declared as mixin procedures. The procedure's first parameter must be of a type that includes a method override for every method referenced through super.

- A mixin procedure can be invoked only as a method. This guarantees that there is no way for the overridden methods of a mixin instance to be accessed from outside the instance.

All rules given in this section can be statically enforced. This is a necessary condition for safety, and for an efficient implementation. These rules are specific to Modula-3, and an extension of another language would certainly differ in many details. However, the basic strategy of generalizing object types (or classes, in other languages) to mixins is fundamental to any such cxtension.

\section{Conclusion}

The inheritance mechanisms in the languages Beta, Smalltalk, and CLOS are representative of three different design choices for inheritance. Although the mechanisms are, on the surface, vcry dissimilar, we identify a common underlying structure. This underlying mechanism combines two sets of attributes such that duplicate attribute definitions are given a value from one set, where the value that is used may refer to the value being eliminated.

Beta and Smalltalk both support single inheritance, in which a single existing definition may be extended with new altributes. In Smalltalk the new attributes may replace existing attributes, which can be accessed directly via super. In contrast, Beta prohibits the extensions from replacing existing attributes; a new definition for an existing attribute has an effect only by being invoked when the original attribute executes the inner command. These two mechanisms have inverse relationships between inherited definition and extensions: the Smalltalk subclass/superclass relationship is analogous to the superpattern/subpattern relationship in Beta, where super is analogous to inner.

CLOS supports multiple inheritance, in which several existing definitions may be combined together. 'To avoid duplication of components, CLOS linearizes the set of primitive components in the inherited definitions. 'This linear list of components is then combined together by the same mechanism underlying Smalltalk and Beta: attribute values appearing earlier in the list replace (and may refer to) those appearing later. One disadvantage of linearization is that the relationships between primitive components may be changed. However, we show that linearization is the basis for the useful technique of mixin programming.

We propose that the underlying inheritance mechanism, which appears in two different restricted forms in Beta and Smalltalk, and is hidden behind linearization in CLOS, be used as the foundation for a general inheritance construct. In this formulation, mixins become the basic definitional construct, and inheritance is interpreted as mixin composition. Since the composition of mixins is explicit, the problem of linearization violating encapsulation does not arise.

It does not appear difficult to extend Beta and Smalltalk to support mixins and generalized inheritance. This work could be applied to CLOS, which already supports mixins, to make them more explicit and 
less susceptible to encapsulation problems. A sketch of an extension to Modula-3 illustrates a possible design for mixins and generalized inheritance.

\section{References}

[1] Cardelli, L. A semantics of multiple inheritance. In Semantics of Data Types (1984), vol. 173 of Lecture Notes in Computer Science, Springer-Verlag, pp. 51-68.

[2] Cardelli, L., Donahue,'J., Glassman, L., Jordan, M., Kalsow, B., and Nelson, G. Modula-3 report (revised). Tech. Rep. 52, Digital Equipment Corporation Systems Research Center, Dec. 1989.

[3] Cook, W. A Denotational Semantics of Inheritance. PhD thesis, Brown University, 1989.

[4] Cook, W., and Palsberg, J. A denotational semantics of inheritance and its correctness. In Proc. of ACM Conf, on Object-Oriented Programming, Systems, Languages and Applications (1989), pp. 433-444.

[5] Dahl, O.-J., Myhrhaug, B., and Nygaard, K. The SIMULA 67 common base language. Tech. rep., Norwegian Computing Center, Oslo, Norway, 1970. Publication S-22.

[6] DeMichiel, L., and Gabriel, R. The Common Lisp Object System: An overview. In European Conference on Object-Oriented Programming (June 1987), pp. 151-170.

[7] Ducournau, R., and Habib, M. On some algorithms for multiple inheritance in object-oriented programming. In European Conference on ObjectOriented Programming (1987), pp. 243-252.

[8] Goldberg, A., And Robson, D. Smalltalk-80: the Language and Its Implementation. AddisonWesley, 1983.

[9] Keene, S. E. Object-Oriented Programming in Common Lisp. Addison-Wesley, 1989.

[10] Kristensen, B. B., Madsen, O. L., MollerPedersen, B., and Nygaard, K. The Beta programming language. In Research Directions in Object-Oriented Programming. MIT Press, 1987, pp. 7-48.

[11] Kristensen, B. B., Madsen, O. L., MollerPederson, B., And Nygaard, K. The Beta programming language - a Scandinavian approach to object-oriented programming, Oct. 1989. OOPSLA Tutorial Notes.
[12] Kristensen, B. B., Madsen, O. L., MollerPendersen, B., ANd Nygaard, K. Abstraction mechanisms in the Beta programming language. Information and Control (1983).

[13] Moon, D. A. Object-oriented programming with Flavors. In Proc. of ACM Conf. on Object-Oriented Programming, Systems, Languages and Applications (1986), pp. 1-8.

[14] Papazoglou, M. P., Georgiadis, P. I., and Maritsas, D. G. An outline of the programming language Simula. Compuler Language 9, 2 (1984), 107-131.

[15] Reddy, U. S. Objects as closures: Abstract semantics of object-oriented languages. In Proc. ACM Conf. on Lisp and Functional Programming (1988), pp. 289-297.

[16] SNYDER, A. Encapsulation and inheritance in object-oriented programming languages. In Proc. of ACM Conf. on Object-Oriented Programming, Systems, Languages and Applications (1986), pp. 38-45.

[17] Snyder, A. Inheritance and the development of encapsulated software systems. In Research Directions in Object-Oriented Programming. MIT Press, 1987, pp. 165-188.

[18] Wand, M. Type inference for record concatenation and multiple inheritance. In Proc. IEEE Symposium on Logic in Computer Science (1989), pp. 9297.

[19] Wegner, P., AND ZdoniK, S. B. Inheritance as a mechanism for incremental modification. In European Conference on Object-Oriented Programming (1988), pp. 55-77. 